\title{
Cognitive Health and Brain Plasticity due to Physical Exercise during Ageing
}

\section{Trevor Archer*}

Department of Psychology, University of Gothenburg, Gothenburg, Sweden

\section{Introduction}

Several aspects concerning the loss of vascular and metabolic integrity that are associated with the ageing process cause wideranging impairments in regional, biomarker and functional domains. For example, the progressive white matter injury arising from a prevailing vascular pathophysiology incorporates both normal ageing and neurodegenerative features of dementia, as well as microvascular brain injury, which originates from a condition of chronic recurrent white matter ischemia that induces oxidative stress and an elevation in the number of total oligodendrocyte lineage cells. Subsequently, perturbances in vascular-induced cognitive deficit and dementia are expressed in the selectively-impaired vasodilator functions of white matter-penetrating arterioles in microvascular brain injury. These perturbances occurs in association with aberrant differentiation of oligodendrocyte progenitor cells as the result of white matter injury; these notions imply simply that myelination maturational processes are associated with perturbations in the maturation of myelinating oligodendrocyte cells [1]. Clinically-relevant improvements in insulin resistance/glucose homeostasis within different populations of ageing adults were observed to be related to the augmentation of cognitive functioning resulting from programs of exercise/activity [2]. Amongst a group of older, elderly adults (mean age $=72.6$ years), expressions of adherence to compliance for exercise were in favour of social dancing exercise, especially regarding its attractiveness for promoting cognitive and social functioning and several indicators of health and well-being [3]. The presence of depression-related problems among the aged has been well-established with cognitive deficits among the more serious health burdens: in this regard, exercise training, bestowing pro-resilience and anti-depressant effects, produces beneficial effects on stress-related mental disorders, indicative of clinical potential. For example, acute physical exercise produces marked increments in the individual alpha peak frequency of subjects offering a cortical parameter associated with neural information processing speed [4]. Further, the effects of exercise upon mood states remains an invariably favourable process, independent of the period of exercise duration or the recovery-from-exercise period [5]. Taken together the consensus of burgeoning evidence is that physical exercise may improve, but most certainly delay, the negative influences of ageing and Azheimer's disease [6].

Physical exercise compliance is promoted increasingly as the major onslaught against the implacableness of age-related declines in physical and cognitive health among older and elder older adults, despite a certain sparsity for reliable indications for the relative efficacies of aerobic, endurance as opposed to resistance, heavy regimes. As a barometer condition for cognitive health, it has been demonstrated repeatedly that the exercise process induces numerous positive effects upon brain functioning, not least through angiogenetic mobilisation of nutrient logistics, as demonstrated by enhanced executive cognitive and working memory functioning, possibly arising from the neurophysiological adaptations through increments in physical fitness independent of any specific type of exercise regime. Thus, among the aged, physical exercise of most different variations enhances cognitive performances, promote brain plasticity, through release of brainderived neurotrophic factor (BDNF), and enhance brain health [7-9], and both human and animal laboratory studies attest to the singular role of BDNF in mediating the effects of exercise upon cognition [10]. Nevertheless, from the perspective of the evidence, discussed above, with the circling type of exercise, there is available a multitude of indications are that high-intensity interval training is a potent stimulus to promote cardiovascular fitness that relates markedly to cognitive performance. In a comparison of sixty-seven older participants (aged 55-75 years) assigned to 16-weeks of either: (i) resistance training, (ii) high-intensity aerobic interval training, (iii) moderate continuous aerobic training and (iv) a control condition, that were tested on the Strops task for executive functioning, and with the Timed-Up-andGo (TUG) procedures and 'submaximal' Bruce treadmill tests for physical functioning, it was observed that the combination of resistance training and high-intensity aerobic interval training provoked superior performance on the enhancement of older individuals' executive cognitive function than the moderate continuous aerobic training with the high-intensity aerobic interval training proving the most beneficial for improvement in information processing speed, providing the greatest gains regarding physical function [11].

In this regard, it has been shown repeatedly that simultaneous exercise-training interventions, especially those applying multimodal exercise programs together with auxiliary tasks that regulated through sensory cues, have promoted cognitive health remarkably well in both healthy older adults and clinically-affected, neurodegenerative patients, e.g. bearing Alzheimer's disease [12]. In an animal model of Alzheimer's disease, physical exercise, through treadmill-running (five sessions per week over four weeks), enhanced protein levels of AMPK-activated protein kinase activity and induced an up-regulation of the peroxisome proliferator-activated receptor gamma co-activator 1 alpha, fibronect in type III domain-containing 5 , and the brain-derived neurotropic pathway thereby instigating and mediating the beneficial effects of exercise on amyloid $\beta$-induced learning and memory impairments [13]. Post-operative cognitive decline may be afflictive among more than ten percent of surgical patients and may induce markedly higher expressions of risk factors associated with advanced age, perioperative infections, and specific metabolic conditions that include obesity and insulin resistance. The rat model of metabolic syndrome, i.e., the "low capacity runner" rats differ 10-fold in their aerobic exercise capacity from the "high capacity runner" rats, the former of which, post-operatively, demonstrate an exaggerated cognitive decline both at three days and at three months of testing; this effect was prevented by pre-operative exercise training [14]. Over several instances of

*Corresponding author: Trevor Archer, Department of Psychology, University of Gothenburg, Gothenburg, Sweden, Tel: +46 31 7864694; E-mail: trevor.archer@ psy.gu.se

Received: January 16, 2018; Accepted: February 07, 2018; Published: February 15,2018

Citation: Archer T (2018) Cognitive Health and Brain Plasticity due to Physical Exercise during Ageing. Clin Exp Psychol 4: 183. doi: 10.4172/2471-2701.1000183

Copyright: (c) 2018 Archer T. This is an open-access article distributed under the terms of the Creative Commons Attribution License, which permits unrestricted use, distribution, and reproduction in any medium, provided the original author and source are credited. 
Citation: Archer T (2018) Cognitive Health and Brain Plasticity due to Physical Exercise during Ageing. Clin Exp Psychol 4: 183. doi: 10.4172/24712701.1000183

Page 2 of 2

cognition and learning, the mediatory effects of BDNF are persistent in the establishment of cellular plasticity $[15,16]$ and are reliably achieved through various forms of physical exertion [17]. Suitably, the characteristics, impact, and scalability of these exercise programs have been documented [18]. Among the diversity of executive functions, controlled behavioral inhibition benefits more selectively from physical exercise than the other executive functions [19]. The epigenetic relationships arising from exercise predispositions gain ever-greater prevalence and frequency, even in the context of cognitive expressions. In the performance of this specific type of behavioral inhibition expression, it appears that the presence of BDNFVal66Val and Val66Met polymorphisms interacted variably with physical exercise parameters on controlled behavioral inhibition performance since Valcarriers without exercise exhibited poorer executive functions performance at age 75 years than their comparison groups with higher exercise levels [20]. In a further study of inhibitory behavior performance, it was shown that the BDNFVal66Met polymorphism interacted with exercise such that inactive Val-homozygous participants, 114 healthy elderly volunteers, with a mean age of 71.53 years, demonstrated a lower inhibition performance than active Val homozygotes and inactive Met carriers [21]. Loss of telomeric proteins, oxidative stress, and hypoxia, contribute to shortening of telomere length generally and cardiac telomere length particularly and disruption of heart function whereas antioxidants, calorie restriction, and exercise can prevent both cardiac telomere attrition and the progression of chronic heart disease [22].

\section{Conclusion}

The inevitable, sacrosanct aspect of the progression of ageing, whether examined at cellular, tissue, organ, epigenetic or functional level, must be endured, and even endorsed, if individuals are to harvest the profits from self-developed opportunities for optimal health manifestations, rather that the neuro generative scenario that generally accompanies ageing. There is an upsurge of findings from various domains posit that the engagement and commitment to healthy lifestyle behaviors, such as physical exercise program, cognitive and social participation, stress reduction, and resilience training, may both prevent and protect against illnesses and disease states that accumulate ageing and concomitantly promote psychological and somatic health.

\section{References}

1. Bagi Z, Brandner DD, Le P, McNeal DW, Gong X, et al. (2017) Vasodilator dysfunction and oligodendrocyte dysmaturation in aging white matter. Ann Neurol.

2. Zhao RR, O'Sullivan AJ, Fiatarone Singh MA (2018) Exercise or physical activity and cognitive function in adults with type 2 diabetes, insulin resistance or impaired glucose tolerance: A systematic review. Eur Rev Aging Phys Act 15: 1.

3. Thøgersen-Ntoumani C, Papathomas A, Foster J, Quested E, Ntoumanis N (2017) 'Shall we dance'? older adults' perspectives on the feasibility of a dance intervention for cognitive function. J Aging Phys Act 28: 1-25.

4. Gutmann B, Zimmer P, Hülsdünker T, Lefebvre J, Binnebößel S, et al. (2018) The effects of exercise intensity and post-exercise recovery time on cortical activation as revealed by EEG alpha peak frequency. Neurosci Lett 668: 159163.
5. Crush EA, Frith E, Loprinzi PD (2018) Experimental effects of acute exercise duration and exercise recovery on mood state. J Affect Disord 229: $282-287$.

6. Panza GA, Taylor BA, MacDonald HV, Johnson BT, Zaleski AL, et al. (2018) Can exercise improve cognitive symptoms of alzheimer's disease? A metaanalysis. J Am Geriatr Soc.

7. de Assis GG, de Almondes KM (2017) Exercise-dependent BDNF as a modulatory factor for the executive processing of individuals in course of cognitive decline. a systematic review. Front Psychol 8: 584.

8. Müller P, Rehfeld K, Schmicker M, Hökelmann A, Dordevic M, et al. (2017) Evolution of neuroplasticity in response to physical activity in old age: the case for dancing. Front Aging Neurosci 9: 56.

9. Vanzella C, Neves JD, Vizuete AF, Aristimunha D, Kolling J, et al. (2017)Treadmill running prevents age-related memory deficit and alters neurotrophic factors and oxidative damage in the hippocampus of Wistar rats. Behav Brain Res 334: 78-85.

10. Chieffi S, Messina G, Villano I, Messina A, Valenzano A et al. (2017) Neuroprotective effects of physical activity: evidence from human and animal studies. Front Neurol 8: 188.

11. Coetsee C, Terblanche $E$ (2017) The effect of three different exercise training modalities on cognitive and physical function in a healthy older population. Eur Rev Aging Phys Act 14: 13.

12. Tait JL, Duckham RL, Milte CM, Main LC, Daly RM (2017) Influence of sequential vs. simultaneous dual-task exercise training on cognitive function in older adults. Front Aging Neurosci 9: 368.

13. Azimi M, Gharakhanlou R, Naghdi N, Khodadadi D, Heysieattalab S (2018) Moderate treadmill exercise ameliorates amyloid- $\beta$-induced learning and memory impairment, possibly via increasing AMPK activity and upregulation of the PGC-1 $\alpha /$ FNDC5/BDNF pathway. Peptides.

14. Feng X, Uchida Y, Koch L, Britton S, Hu J, et al. (2017) Exercise prevents enhanced postoperative neuroinflammation and cognitive decline and rectifies the gut microbiome in a rat model of metabolic syndrome. Front Immunol 8 : 1768

15. Dong J, Zhao J, Lin $\mathrm{Y}$, Liang $\mathrm{H}$, He X, et al. (2018) Exercise improves recognition memory and synaptic plasticity in the prefrontal cortex for rats modelling vascular dementia. Neurol Res 40: 68-77.

16. Gomes-Osman J, Cabral DF, Hinchman C, Jannati A, Morris TP, et al. (2017) The effects of exercise on cognitive function and brain plasticity - a feasibility trial. Restor Neurol Neurosci 35: 547-556.

17. Walsh JJ, Bentley RF, Gurd BJ, Tschakovsky ME (2017) Short-duration maximal and long-duration submaximal effort forearm exercise achieve elevations in serum brain-derived neurotrophic factor. Front Physiol 8: 746.

18. Balis LE, Strayer T, Ramalingam N, Wilson M, Harden SM (2018) Openaccess physical activity programs for older adults: a pragmatic and systematic review. Gerontologist.

19. Gajewski PD, Falkenstein M (2017) Physical activity and neurocognitive functioning in aging - a condensed updated review. Eur Rev Aging Phys Act 13: 1 .

20. Thibeau S, McFall GP, Wiebe SA, Anstey KJ, Dixon RA (2016) Genetic factors moderate everyday physical activity effects on executive functions in aging: evidence from the victoria longitudinal study. Neuropsychology 30: 6-17.

21. Canivet $A$, Albinet $C T$, Rodríguez-Ballesteros $M$, Chicherio $C$, Fagot $D$, et al. (2017) Interaction between bdnf polymorphism and physical activity on inhibitory performance in the elderly without cognitive impairment. Front Hum Neurosci 11: 541.

22. Booth SA, Charchar FJ (2017) Cardiac telomere length in heart development function, and disease. Physiol Genomics 49: 368-384. 